\title{
Characteristics of European adults who dropped out from the Food4Me Internet-based personalised nutrition intervention
}

\author{
Katherine M Livingstone ${ }^{1}$, Carlos Celis-Morales ${ }^{1}$, Anna L Macready ${ }^{2}$, Rosalind Fallaize ${ }^{2}$, \\ Hannah Forster ${ }^{3}$, Clara Woolhead ${ }^{3}$, Clare B O'Donovan ${ }^{3}$, Cyril FM Marsaux ${ }^{4}$, \\ Santiago Navas-Carretero ${ }^{5,6}$, Rodrigo San-Cristobal ${ }^{6}$, Silvia Kolossa ${ }^{7}$, Lydia Tsirigoti ${ }^{8}$, \\ Christina P Lambrinou ${ }^{8}$, George Moschonis ${ }^{8}$, Agnieszka Surwiłło9, Christian A Drevon ${ }^{10,}$ \\ Yannis Manios ${ }^{8}$, Iwona Traczyk ${ }^{9}$, Eileen R Gibney ${ }^{3}$, Lorraine Brennan ${ }^{3}$, \\ Marianne C Walsh ${ }^{3}$, Julie A Lovegrove ${ }^{2}$, J Alfredo Martinez ${ }^{6}$, Wim HM Saris ${ }^{4}$, \\ Hannelore Daniel ${ }^{7}$, Mike Gibney ${ }^{3}$ and John C Mathers ${ }^{1, *}$ on behalf of the Food 4 Me Study \\ 'Human Nutrition Research Centre, Institute of Cellular Medicine, Newcastle University, Biomedical Research \\ Building, Campus for Ageing and Vitality, Newcastle upon Tyne, NE4 5PL, UK: ${ }^{2}$ Hugh Sinclair Unit of Human \\ Nutrition and Institute for Cardiovascular and Metabolic Research, University of Reading, Reading, UK: ${ }^{3} U C D$ \\ Institute of Food and Health, University College Dublin, Dublin, Republic of Ireland: ${ }^{4}$ Department of Human Biology, \\ NUTRIM School of Nutrition and Translational Research in Metabolism, Maastricht University Medical Centre, Maastricht, \\ The Netherlands: ${ }^{5}$ Center for Nutrition Research, University of Navarra, Pamplona, Spain: ${ }^{6} \mathrm{CIBER}$ Fisiopatología \\ Obesidad y Nutrición (CIBERobn), Instituto de Salud Carlos III, Madrid, Spain: ${ }^{7}$ ZIEL Research Center of Nutrition and \\ Food Sciences, Biochemistry Unit, Technical University of Munich, Munich, Germany: ${ }^{8}$ Department of Nutrition and \\ Dietetics, Harokopio University, Athens, Greece: ${ }^{9}$ National Food \& Nutrition Institute (IZZ), Warsaw, Poland: \\ ${ }^{10}$ Department of Nutrition, Institute of Basic Medical Sciences, Faculty of Medicine, University of Oslo, Oslo, Norway
}

Submitted 14 January 2016: Final revision received 22 June 2016: Accepted 28 June 2016: First published online 5 August 2016

\begin{abstract}
Objective: To characterise participants who dropped out of the Food4Me Proof-ofPrinciple study.

Design: The Food4Me study was an Internet-based, 6-month, four-arm, randomised controlled trial. The control group received generalised dietary and lifestyle recommendations, whereas participants randomised to three different levels of personalised nutrition (PN) received advice based on dietary, phenotypic and/or genotypic data, respectively (with either more or less frequent feedback). Setting: Seven recruitment sites: UK, Ireland, The Netherlands, Germany, Spain, Poland and Greece.

Subjects: Adults aged 18-79 years ( $n$ 1607).

Results: A total of 337 (21\%) participants dropped out during the intervention. At baseline, dropouts had higher BMI $\left(0.5 \mathrm{~kg} / \mathrm{m}^{2} ; P<0.001\right)$. Attrition did not differ significantly between individuals receiving generalised dietary guidelines (Control) and those randomised to PN. Participants were more likely to drop out (OR; 95\% $\mathrm{CI})$ if they received more frequent feedback $(1 \cdot 81 ; 1.36,2 \cdot 41 ; P<0 \cdot 001)$, were female $(1 \cdot 38 ; 1 \cdot 06,1 \cdot 78 ; P=0.015)$, less than 45 years old $(2 \cdot 57 ; 1 \cdot 95,3 \cdot 39 ; P<0 \cdot 001)$ and obese $(2 \cdot 25 ; 1 \cdot 47,3 \cdot 43 ; P<0 \cdot 001)$. Attrition was more likely in participants who reported an interest in losing weight $(1.53 ; 1.19,1.97 ; P<0.001)$ or skipping meals $(1.75 ; 1.16,2.65 ; P=0.008)$, and less likely if participants claimed to eat healthily frequently $(0.62 ; 0.45,0.86 ; P=0.003)$.

Conclusions: Attrition did not differ between participants receiving generalised or PN advice but more frequent feedback was related to attrition for those randomised to PN interventions. Better strategies are required to minimise dropouts among younger and obese individuals participating in PN interventions and more frequent feedback may be an unnecessary burden.
\end{abstract}

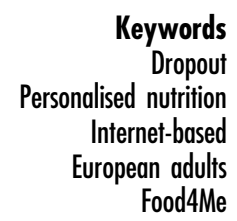

Dropout

Internet-based Food4Me
Improving diet and physical activity behaviours is an important means of lowering the risk of noncommunicable diseases, promoting healthy ageing and increasing well-being ${ }^{(1,2)}$. Given that the burden of ill-health is increasing ${ }^{(1,3)}$, alternative strategies for improving dietary behaviours based on predictive, 
personalised, preventive and participatory interventions may be more effective than conventional 'one size fits all' generalised dietary advice ${ }^{(4,5)}$. Personalised nutrition (PN) may be a more effective approach for improving dietary and physical activity behaviours than non-personalised advice $^{(5-7)}$. However, the relevance of the outcomes of PN interventions may be limited if there are systematic sociodemographic or behavioural differences between study completers and dropouts, which may result in specific target groups (e.g. obese individuals) not benefiting from PN. Sociodemographic variables such as age, social class, occupation and financial factors are key determinants of dropouts in lifestyle-based interventions ${ }^{(8,9)}$, with more recent evidence also suggesting that behavioural characteristics are important predictors of attrition $^{(10)}$. Dropouts from dietary and lifestyle interventions may differ considerably from one intervention to another $^{(8)}$, with approximately a third of participants dropping out of weight-loss interventions ${ }^{(11-14)}$ and $20 \%$ from other diet and lifestyle interventions ${ }^{(8,15)}$. For reasons of cost-effectiveness, reach and scalability, Internet-based lifestyle interventions are increasingly popular ${ }^{(16,17)}$ although more information is needed on the characteristics of dropouts from such studies. Understanding the determinants of attrition from Internet-based PN intervention studies will inform the design of more efficiently targeted lifestyle interventions.

The aim of the present paper was to characterise participants who dropped out of the Food4Me Proofof-Principle Internet-based trial of PN, which was designed to improve dietary and physical activity behaviours. Sociodemographic, anthropometric, dietary, behavioural and health-related characteristics were compared between completers and those who dropped out.

\section{Methods}

\section{Study design}

The Food4Me Proof-of-Principle study was a 6-month, four-arm, Internet-based, randomised controlled trial conducted across seven European countries via www. food $4 \mathrm{me}$.org ${ }^{(18)}$. The randomised controlled trial was designed to emulate a real-life Internet-based PN service and aimed to investigate: (i) whether personalisation of dietary advice assists and/or motivates participants to eat a healthier diet in comparison with non-personalised, conventional healthy eating guidelines; and (ii) whether personalisation based on individualised phenotypic or genotypic information is more effective in assisting and/or motivating study participants to make, and to sustain, appropriate healthy changes than personalisation based on diet alone. The Research Ethics Committees at each University or Research Centre delivering the intervention granted ethical approval for the study. The Food4Me trial was registered as a randomised controlled trial
(NCT01530139) at Clinicaltrials.gov. All participants expressing an interest in the study were asked to sign online consent forms at two stages in the screening process.

\section{Recruitment and eligibility criteria}

Participants were recruited via the Internet to emulate an Internet-based PN service. This was aided by local and national advertising of the study via the Internet, radio, newspapers, posters, e-flyers, social media and word of mouth. Recruitment sites were as follows: University College Dublin (Ireland), Maastricht University (The Netherlands), University of Navarra (Spain), Harokopio University (Greece), University of Reading (UK), National Food and Nutrition Institute (Poland) and Technical University of Munich (Germany). Participants were excluded if they were: $<18$ years of age; pregnant or lactating; had no or limited access to the Internet; were following a prescribed diet for any reason, including weight loss, in the last 3 months; or had diabetes, coeliac disease, Crohn's disease, or any metabolic disease or condition altering nutritional requirements such as thyroid disorders (if condition was not controlled), allergies or food intolerances. Participants were incentivised to join the study by receiving a personalised feedback report at month 6 based on their dietary, phenotypic and genotypic information, regardless of their treatment arm allocation.

\section{Intervention arms}

A total of 1607 participants were randomised to one of four intervention arms. Participants received non-personalised, generalised dietary and physical activity (PA) advice (Control) or one of three levels of PN: Level 1 (L1), based on personal current PA plus diet alone; Level 2 (L2), based on PA plus dietary and phenotypic data; Level 3 (L3), based on PA plus dietary, phenotypic and genotypic data. Participants randomised to L1, L2 or L3 were further randomised into 'low intensity' or 'high intensity' intervention groups. Participants in the low intensity group received personalised feedback three times during the intervention (at baseline, month 3 and month 6), whereas those randomised to the high intensity group received personalised feedback five times during the intervention (at baseline and months 1, 2, 3 and 6). In addition, the high intensity group had access to an online forum for discussion of topics related to the intervention, personalised recipes and had more personalised feedback on PA. Further details of the Food4Me Proofof-Principle study are provided elsewhere ${ }^{(18)}$.

\section{Personalised feedback report}

At baseline, month 3 and month 6, intakes of five food groups (fruits and vegetables, whole grains, low-fat dairy products, oily fish, and red meat and processed meat) and seventeen nutrients were categorised as too high or too 
low for each participant randomised to PN. Contributing foods were identified and specific messages were developed, according to standardised algorithms, to advise change in intake of those foods. For participants randomised to L2 and L3, feedback also included phenotypic measures (L2) and phenotypic and genotypic data (L3) ${ }^{(18)}$.

\section{Screening questionnaires and dietary intakes}

Individuals who were interested in participating in the study completed an online screening questionnaire to collect information on sociodemographic, health and anthropometric characteristics. This questionnaire also included information on dietary habits (e.g. meal skipping) and reasons for interest in participation in the study (e.g. weight loss). Likert scale responses were aggregated into three categories: 'disagree' ('completely disagree' and 'disagree'), 'neither disagree nor agree' and 'agree' ('agree' and 'completely agree'); and questions relating to frequency of the occurrence into two categories: 'often' ('every day' and ' $4-6$ times per week') and 'rarely' (' $1-3$ times per week' and '(almost) never'; see online supplementary material, Supplemental Table 1).

Participants were asked to complete an online FFQ to estimate usual dietary intake at screening, baseline (month 0 ) and at months 3 and 6 (also at months 1 and 2 for the high intensity group only). This FFQ was developed and validated for the Food4Me study ${ }^{(19,20)}$, and included 157 food items consumed frequently in each of the seven recruitment countries. Intakes of foods, total energy and macronutrients were computed in real time using a food composition database based on McCance and Widdowson's The Composition of Foods ${ }^{(21)}$. BMR was estimated using the Oxford equation ${ }^{(22)}$. Intakes were assessed using standardised recommendations ${ }^{(18)}$ for foods and food groups that were integrated and harmonised across eight European countries (UK, Ireland, Germany, The Netherlands, Spain, Greece, Poland and Norway) ${ }^{(23-26)}$. The following five food group recommendations were used in the present analysis: (i) eat at least five portions of fruits and vegetables every day (operationalised as $\geq 400 \mathrm{~g}$ ); (ii) eat at least three portions of wholegrain products daily ( $\geq 50 \mathrm{~g}$ ); (iii) eat at least three portions of low-fat dairy products daily ( $\geq 600 \mathrm{~g}$ ); (iv) eat at least one portion of oily fish per week $(\geq 150 \mathrm{~g})$; and (v) eat fewer than three portions of red meat and processed meat per week $(\leq 450 \mathrm{~g})^{(18)}$.

\section{Sociodemographic and health-related measures}

Body weight, height and waist circumference were selfmeasured and self-reported. BMI was estimated from body weight and height. Self-reported measurements were validated in a sub-sample of the participants ( $n$ 140) and showed a high degree of reliability ${ }^{(27)}$. Participants were sent finger-prick based Dry Blood Spot cards (collected five drops equivalent to $150 \mu \mathrm{l}$ of blood per card) which were completed and returned by post to recruitment centres and used to estimate blood total cholesterol concentrations. PA level and time spent in sedentary behaviour were estimated from triaxial accelerometers (TracmorD, Philips Consumer Lifestyle, The Netherlands). Participants self-reported smoking habits and occupation. Based on European classifications of occupations the following groupings were used: 'Professional and managerial' (professionals; managers); 'Intermediate' (armed forces occupations; technicians and associate professionals; clerical support workers); and 'Routine and manual' (craft and related trades workers; plant and machine operators and assemblers; service and sales workers; elementary occupations; skilled agricultural, forestry and fishery workers) ${ }^{(28,29)}$. Categories for 'Students' and 'Retired and unemployed' were added. See the online supplementary material, 'Supplementary Methods' for further information on the study design.

\section{Statistical analyses}

Data were analysed using the statistical software package Stata version 13. Screening data (dietary habits, FFQ, reasons for interest in the study, ethnicity, medication use and health characteristics) plus measurements of waist circumference, sedentary behaviour and PA level, which were collected at baseline, were used in the present analysis. Logistic regression and multiple linear regression were used to test for significant differences between categorical and continuous variables, respectively. The odds ratio for dropping out before month 6 was estimated for categorical variables. All analyses were adjusted for baseline age, sex and country. PA outcomes were further adjusted for time spent wearing the accelerometer and season. Sensitivity analyses were performed to estimate odds ratios for dropping out at the interim time point (month 3). Results were deemed significant at $P<0 \cdot 05$.

\section{Results}

A total of 1607 participants were randomised into the study at baseline. As summarised in Fig. 1, 337 participants (21\%) dropped out and 1270 participants completed the 6-month intervention period. Of the 337 participants who dropped out, 127 (38\%) dropped out before completing baseline measurements and a total of 291 (86\%) had dropped out by month 3 (Fig. 1).

\section{Health and lifestyle-related characteristics}

Dropouts were on average 6 years younger than completers and were predominantly female (Table 1 ). In addition, dropouts weighed more, had higher BMI and lower waist circumference (Table 1). More participants who dropped out of the study (8\%) than those who completed it reported being interested in participating 


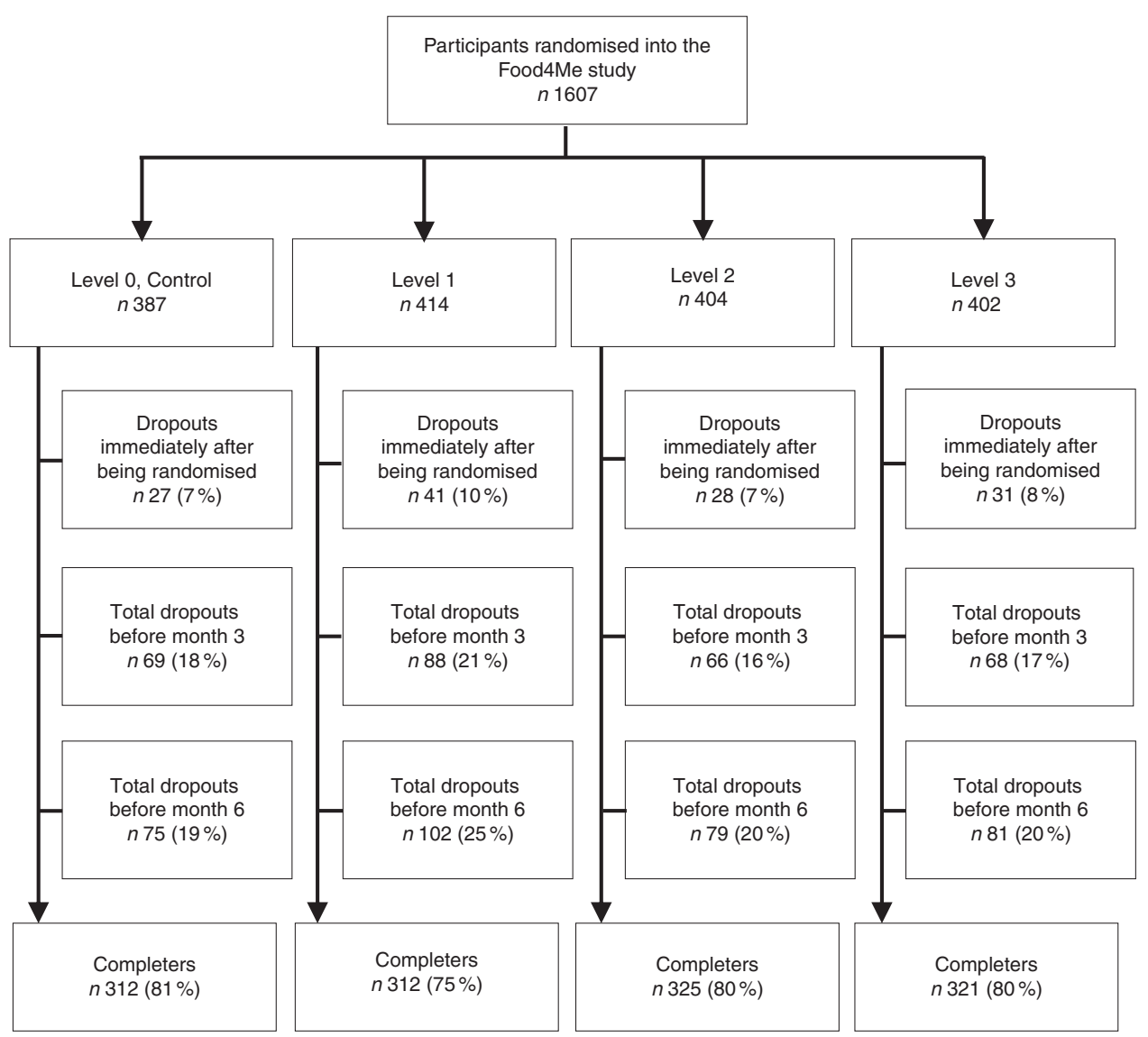

Fig. 1 Flow diagram of cumulative dropouts from the Food4Me Proof-of-Principle study

because they wanted to lose weight. No significant differences in occupation classification were observed between completers and those who dropped out. Furthermore, there were no significant differences in height, PA level, sedentary behaviour or total cholesterol concentration between groups. The percentage of individuals following a restricted diet, taking medication or presenting with clinically diagnosed diseases did not differ significantly between completers and dropouts (Table 1).

\section{Dietary characteristics}

No significant differences in total energy intake or ratio of energy intake to BMR were identified between individuals who completed the 6-month intervention and those who dropped out (Table 2). Completers reported consuming more energy from PUFA and less salt than dropouts. Percentage energy intakes from total fat, SFA and MUFA, protein and carbohydrate were not significantly different between dropouts and completers (Table 2). The percentage of individuals who met the dietary recommendations for oily fish, whole grains, red meat, fruit and vegetables, and low-fat dairy products did not differ significantly between completers and dropouts (Table 2).

\section{OR of dropping out by intervention arm}

Attrition did not differ significantly depending on whether individuals were randomised to receive generalised dietary guidelines (Control) or any level of PN (L1, L2 or L3; Table 3). When levels of $\mathrm{PN}$ were grouped together (L1, L2 and L3), there was no significant difference in OR for dropping out between participants who received generalised dietary advice (Control) and those who received PN advice (Table 3 ). However, when intervention arms were grouped according to whether individuals received high or low intensity feedback, the odds of participants dropping out were higher in those randomised to receive high intensity feedback than low intensity feedback $(\mathrm{OR}=1 \cdot 81 ; 95 \%$ CI $1 \cdot 36,2 \cdot 41$; $P<0 \cdot 001)$.

\section{OR of dropping out by sociodemographic and dietary characteristics}

Stratification by age revealed that the odds of participants dropping out were higher if they were under 45 years of age than if they were over 45 years (Table 4). In addition, the odds of females dropping out were higher than for males. Compared with normal-weight individuals, the odds of dropping out were higher in obese individuals. 
Table 1 Baseline sociodemographic characteristics of participants who completed the intervention and those who dropped out by month 6 , Food4Me Proof-of-Principle study

\begin{tabular}{|c|c|c|c|c|c|}
\hline & \multicolumn{2}{|c|}{ Completers ( $n$ 1270) } & \multicolumn{2}{|c|}{ Dropouts (n 337) } & \multirow[b]{2}{*}{$P^{\star}$} \\
\hline & Mean or \% & SD & Mean or \% & SD & \\
\hline Age (years) & $40 \cdot 8$ & $13 \cdot 0$ & 34.8 & $12 \cdot 3$ & $<0.001$ \\
\hline Female (\%) & $57 \cdot 4$ & - & $66 \cdot 8$ & - & 0.017 \\
\hline \multicolumn{6}{|l|}{ Ethnicity (\%) } \\
\hline Caucasian & $96 \cdot 9$ & - & $96 \cdot 1$ & - & 0.83 \\
\hline \multicolumn{6}{|l|}{ Occupation (\%) } \\
\hline Professional and managerial & $40 \cdot 0$ & - & 34.6 & - & 0.53 \\
\hline Intermediate & $26 \cdot 1$ & - & $25 \cdot 5$ & - & 0.98 \\
\hline Routine and manual & 9.5 & - & $11 \cdot 1$ & - & 0.42 \\
\hline Student & $14 \cdot 0$ & - & $21 \cdot 2$ & - & 0.13 \\
\hline Retired & 3.0 & - & $2 \cdot 4$ & - & 0.39 \\
\hline Unemployed & 7.4 & _ & $5 \cdot 3$ & - & 0.88 \\
\hline \multicolumn{6}{|l|}{ Anthropometrics } \\
\hline Body weight (kg) & 74.6 & $15 \cdot 7$ & $75 \cdot 4$ & $17 \cdot 0$ & $<0.001$ \\
\hline BMI $\left(\mathrm{kg} / \mathrm{m}^{2}\right)$ & $25 \cdot 4$ & 4.8 & $25 \cdot 9$ & 5.5 & $<0.001$ \\
\hline Waist circumference $(\mathrm{cm})$ & $85 \cdot 9$ & 13.7 & $84 \cdot 6$ & 14.7 & 0.015 \\
\hline Height $(m)$ & 1.7 & 0.1 & 1.7 & 0.1 & 0.89 \\
\hline \multicolumn{6}{|l|}{ Physical activity } \\
\hline PAL & 1.7 & 0.2 & 1.7 & 0.2 & 0.86 \\
\hline Sedentary behaviour $(\min / \mathrm{d})$ & 747 & $75 \cdot 2$ & 732 & $77 \cdot 1$ & 0.31 \\
\hline \multicolumn{6}{|l|}{ Dietary conditions (\%) } \\
\hline Want to lose weight & $45 \cdot 8$ & - & 53.7 & - & 0.002 \\
\hline Restricted diet & $6 \cdot 7$ & - & $8 \cdot 3$ & - & 0.66 \\
\hline \multicolumn{6}{|l|}{ Medication use (\%) } \\
\hline Prescribed medication & 30.5 & - & $27 \cdot 6$ & - & 0.67 \\
\hline Non-prescribed medication & $10 \cdot 3$ & - & $7 \cdot 7$ & - & 0.32 \\
\hline \multicolumn{6}{|l|}{ Health and disease } \\
\hline Total cholesterol (mmol/l) & 4.6 & $1 \cdot 0$ & $4 \cdot 3$ & 0.9 & 0.06 \\
\hline Current smoker $(\%)$ & $11 \cdot 7$ & - & 13.7 & - & 0.66 \\
\hline Cancer (\%) & 1.6 & - & 0.3 & - & 0.21 \\
\hline High blood pressure (\%) & 7.9 & - & $6 \cdot 8$ & - & 0.21 \\
\hline Heart disease (\%) & 1.4 & - & 1.2 & - & 0.61 \\
\hline Diabetes (\%) & 0.6 & - & 0.6 & - & 0.61 \\
\hline Blood disorders (\%) & $1 \cdot 1$ & - & 0.6 & - & 0.29 \\
\hline
\end{tabular}

PAL, physical activity level.

${ }^{*}$ Multiple linear regression and logistic regression were used to test for significant differences between groups in continuous and categorical variables, respectively. Analyses were adjusted for age, sex and country. Significant $P$ values are indicated in bold.

Attrition was not significantly different in overweight compared with normal-weight individuals, between non-smokers and current smokers, or between individuals with low $v$. high PA level or low $v$. high sedentary behaviour (Table 4).

Compared with the average for all countries, the odds of dropping out were higher in participants from Ireland, whereas the odds in participants from The Netherlands were lower. Attrition was not significantly different for participants from Germany, Greece, Poland, Spain or the UK when compared with the overall average (Table 4). Being in an intermediate or routine/manual occupation, or being a student or retired/unemployed, did not significantly affect the odds of dropping out from the study compared with being in a professional/managerial occupation (Table 4). Baseline diet was not a predictor of dropping out. Attrition did not differ significantly between individuals who met the recommendations for oily fish, whole grains, red meat, fruit and vegetables, and low-fat dairy products compared with those who did not (Table 4).
OR of dropping out by behavioural characteristics As illustrated in Fig. 2, the odds of dropping out were higher in participants who had signed up to the study with the aim of losing weight $(\mathrm{OR}=1.53$; $95 \% \mathrm{CI}$ $1.19, \quad 1.97 ; \quad P<0.001)$. Attrition was not significantly different if participants had, or had not, signed up with the aim of gaining weight, wanting to know what foods are best for them, wishing to improve their own or their family's health, for well-being reasons, or in individuals with an interest in sports performance or preventing a future illness (see online supplementary material, Supplemental Table 2).

Odds of attrition were higher if participants ate their main meal away from home $(\mathrm{OR}=1.33$; $95 \% 1.04$, $1.72 ; P=0.023)$ and higher if they regularly skipped meals (OR $=1.75 ; 95 \%$ CI 1.16, 2.65; $P=0.008$; Fig. 2). Odds for dropping out were not significantly different depending on whether participants prepared a meal from scratch, ate many or few hot meals per day, or spent little time preparing a main meal (Supplemental Table 2). 
Table 2 Baseline dietary characteristics of participants who completed the intervention and those who dropped out by month 6 , Food4Me Proof-of-Principle study

\begin{tabular}{|c|c|c|c|c|c|}
\hline & \multicolumn{2}{|c|}{ Completers ( $n$ 1270) } & \multicolumn{2}{|c|}{ Dropouts (n 337) } & \multirow[b]{2}{*}{$P^{\star}$} \\
\hline & Mean or \% & SD & Mean or \% & SD & \\
\hline \multicolumn{6}{|l|}{ Nutrient intake } \\
\hline Total energy (kJ/d) & 11531 & 5054 & 11699 & 4807 & 0.43 \\
\hline Total energy (kcal/d) & 2756 & 1208 & 2796 & 1149 & 0.43 \\
\hline El:BMR & 1.8 & 0.7 & 1.8 & 0.7 & 0.94 \\
\hline Total fat (\% of energy) & $35 \cdot 5$ & $6 \cdot 5$ & $35 \cdot 1$ & $6 \cdot 5$ & 0.29 \\
\hline SFA (\% of energy) & $14 \cdot 0$ & 3.4 & $14 \cdot 1$ & $3 \cdot 6$ & 0.64 \\
\hline MUFA (\% of energy) & $13 \cdot 6$ & 3.5 & $13 \cdot 2$ & $3 \cdot 2$ & 0.10 \\
\hline PUFA (\% of energy) & $5 \cdot 7$ & 1.5 & 5.4 & $1 \cdot 2$ & 0.002 \\
\hline Protein (\% of energy) & $16 \cdot 9$ & 3.6 & $17 \cdot 1$ & $4 \cdot 1$ & 0.41 \\
\hline Carbohydrate (\% of energy) & $46 \cdot 8$ & $8 \cdot 2$ & $47 \cdot 3$ & $8 \cdot 3$ & 0.70 \\
\hline Sugars (\% of energy) & $21 \cdot 2$ & $6 \cdot 1$ & $21 \cdot 0$ & $6 \cdot 7$ & 0.21 \\
\hline Dietary fibre $(g / d)$ & $33 \cdot 2$ & $18 \cdot 9$ & 33.9 & $20 \cdot 6$ & 0.35 \\
\hline Salt $(g / d)$ & $8 \cdot 1$ & $4 \cdot 2$ & $8 \cdot 6$ & 7.9 & 0.050 \\
\hline \multicolumn{6}{|c|}{ Meeting dietary recommendations (\%) } \\
\hline Oily fish & 34.7 & - & $32 \cdot 3$ & - & 0.92 \\
\hline Whole grains & $77 \cdot 6$ & - & $75 \cdot 7$ & - & 0.74 \\
\hline Red meat & $48 \cdot 0$ & - & $49 \cdot 6$ & - & 0.67 \\
\hline Fruit and vegetables & $57 \cdot 7$ & - & $56 \cdot 4$ & - & 0.66 \\
\hline Low-fat dairy & 8.0 & - & 6.5 & - & 0.29 \\
\hline
\end{tabular}

El:BMR, ratio of energy intake to BMR.

${ }^{*}$ Multiple linear regression and logistic regression were used to test for significant differences between groups in continuous and categorical variables, respectively. Analyses were adjusted for age, sex and country. Significant $P$ values are indicated in bold.

Table 3 Odds of participants dropping out at month 6 by intervention arm, Food4Me Proof-of-Principle study

\begin{tabular}{lccc}
\hline & OR & $95 \% \mathrm{Cl}$ & $P^{\star}$ \\
\hline Control (ref.) $v$. & & & \\
L1 (low and high intensity) & 1.40 & $0.99,1.98$ & 0.05 \\
L2 (low and high intensity) & 1.04 & $0.72,1.48$ & 0.85 \\
L3 (low and high intensity) & 1.07 & $0.75,1.53$ & 0.70 \\
Control (ref.) v. personalised nutrition & 1.17 & $0.87,1.56$ & 0.30 \\
Low (ref.) v. high intensity feedback & 1.81 & $1.36,2.41$ & $<\mathbf{0 . 0 0 1}$ \\
\hline &
\end{tabular}

Odds of dropping out were lower if participants reported that they frequently ate healthily $(\mathrm{OR}=0 \cdot 62 ; 95 \%$ CI $0.45,0.86 ; P=0.003$ ) and lower if they reported eating healthily without having to think about it consciously (OR= 0.74; 95\% CI 0.56, 0.97); $P=0.031$; Fig. 2). Attrition was not significantly different depending on whether participants reported being in control of their health, staying healthy by taking care of themselves, agreed that efforts to improve their health were a waste of time, agreed that there was no use in concerning themselves with their health or felt weird if they did not eat healthily (Supplemental Table 2).

\section{Sensitivity analyses}

Factors predicting the likelihood of dropping out by month 3 were similar to those observed at month 6 . However, odds of early attrition were higher if participants reported having a clinically diagnosed disease (see online supplementary material, Supplemental Table 2). Furthermore, odds of dropping out in overweight individuals were higher by month 3, compared with normal-weight individuals. The odds of dropping out by month 3 were lower in individuals who indicated that they had signed up to the study because they thought it was important to support academic studies, and lower among those who were curious to find out what happened in academic studies (Supplemental Table 2).

\section{Discussion}

The present study is the first to investigate the sociodemographic, anthropometric, dietary, behavioural and health-related characteristics of participants who dropped out of a 6-month Internet-based study of PN. Our main findings suggest that dropouts were more likely to be younger, obese individuals who skipped meals more often 
Table 4 Odds of participants dropping out at month 6 by baseline sociodemographic characteristics and dietary adequacies, Food4Me Proof-of-Principle study

\begin{tabular}{|c|c|c|c|}
\hline & OR & $95 \% \mathrm{Cl}$ & $P^{*}$ \\
\hline Under 45 years (ref.) $v$. over 45 years & 2.57 & $1.95,3.39$ & $<0.001$ \\
\hline Male (ref.) $v$. female & 1.38 & $1.06,1.78$ & 0.015 \\
\hline \multicolumn{4}{|l|}{ BMI category (ref., normal weight) } \\
\hline Overweight & $1 \cdot 31$ & $0.91,1.90$ & $0 \cdot 15$ \\
\hline Obese & $2 \cdot 25$ & $1.47,3.43$ & $<0.001$ \\
\hline Non-smoker (ref.) v. current smoker & $1 \cdot 11$ & $0.86,1.44$ & 0.41 \\
\hline \multicolumn{4}{|l|}{ Country (ref. = overall average) } \\
\hline Germany & 1.09 & $0.76,1.56$ & 0.66 \\
\hline Greece & 0.90 & $0.63,1.27$ & 0.54 \\
\hline Ireland & 1.62 & $1 \cdot 20,2 \cdot 18$ & 0.002 \\
\hline Netherlands & 0.18 & $0.09,0.35$ & $<0.001$ \\
\hline Poland & 1.08 & $0.77,1.50$ & 0.67 \\
\hline Spain & 1.06 & $0.75,1.52$ & 0.73 \\
\hline UK & $1 \cdot 17$ & $0.85,1.62$ & 0.33 \\
\hline \multicolumn{4}{|l|}{ Occupation (ref. = professional and managerial) } \\
\hline Intermediate & 1.08 & $0.73,1.59$ & 0.70 \\
\hline Routine and manual & $1 \cdot 22$ & $0.73,2.08$ & 0.45 \\
\hline Student & 0.73 & $0.45,1.17$ & 0.19 \\
\hline Retired and unemployed & 1.37 & $0.75,2.52$ & 0.31 \\
\hline \multicolumn{4}{|l|}{$\begin{array}{l}\text { Meeting dietary recommendations } \\
\text { (ref. = not meeting recommendation) }\end{array}$} \\
\hline Fruit and vegetables ( $\geq 5$ portions $/ d$ ) & 1.05 & $0.82,1.35$ & 0.69 \\
\hline Whole grains $(\geq 50 \mathrm{~g} / \mathrm{d})$ & 0.93 & $0.70,1.24$ & 0.63 \\
\hline Red meat ( $\leq 3$ servings/week) & 0.93 & $0.72,1 \cdot 20$ & 0.56 \\
\hline Oily fish ( $\geq 1$ serving/week) & 0.99 & $0.77,1.31$ & 0.99 \\
\hline Low-fat dairy products ( $\geq 3$ servings/d) & 0.77 & $0.48,1.26$ & 0.30 \\
\hline
\end{tabular}

ref., reference category.

*Logistic regression was used to test for significant differences between groups. Analyses were adjusted for age, sex and country.

Significant $P$ values are indicated in bold.

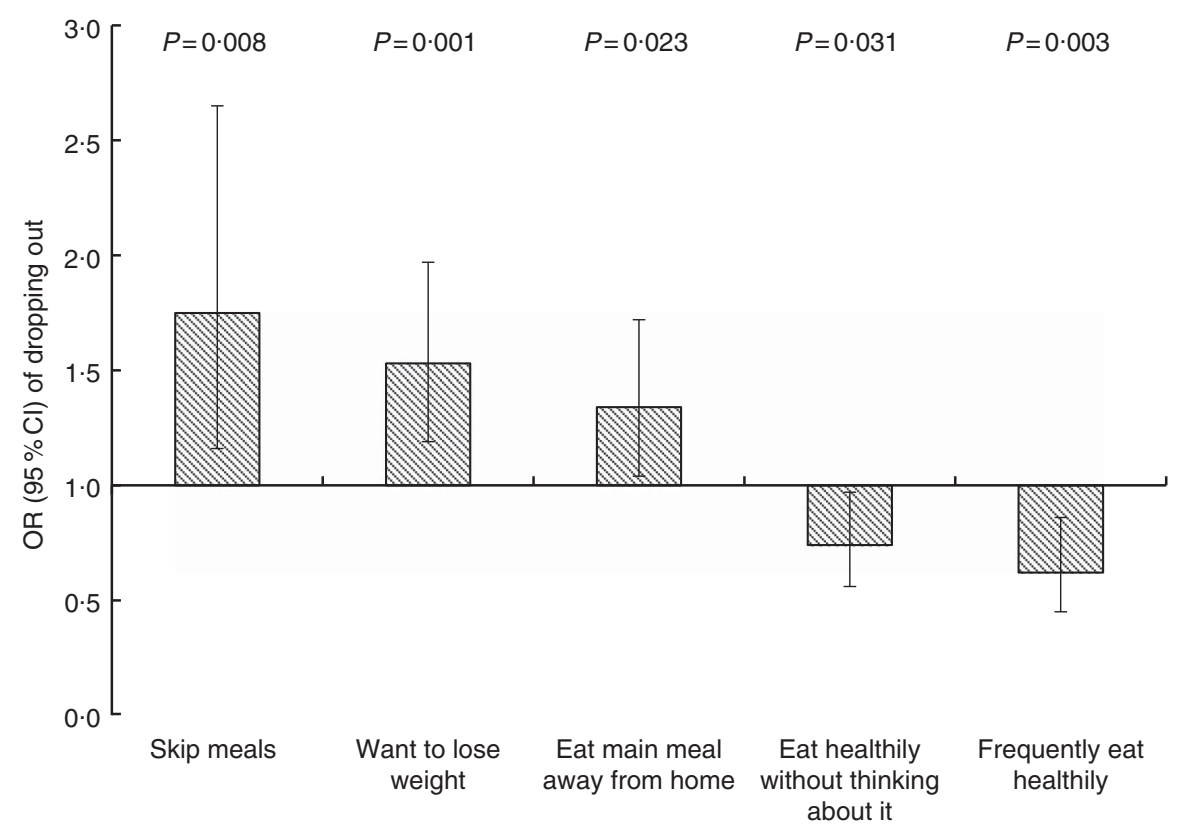

Fig. 2 The odds for participants dropping out according to their dietary behaviours and reasons for participation in the study at baseline, Food4Me Proof-of-Principle study*. Values are adjusted odds ratios, with their $95 \%$ confidence interval represented by vertical bars. *Logistic regression was used to test for significant differences between groups. Models were adjusted for age, sex and country. Variables are dichotomous (reference group is 'no'/'disagree')

and were motivated by weight loss. Furthermore, more frequent data collection and PN feedback increased the likelihood of individuals dropping out.
The dropout rate observed in the present study is well within the range expected from a traditional faceto-face lifestyle intervention of this duration ${ }^{(30)}$. A recent 
meta-analysis on the effectiveness of web-based interventions $^{(31)}$ concluded that web-based interventions were as effective as face-to-face interventions in achieving weight loss and that the dropout rate was $21 \%$, which is similar to the dropout rate in our study. However, the studies included in the meta-analysis were heterogeneous, with dropout rates as high as $40 \%^{(32,33)}$. Our findings suggest that individuals interested in joining the Food4Me study for the purpose of losing weight were more likely to drop out. The present study was not designed, or advertised, as a weight-loss study, but rather as a PN intervention aiming to improve diet and physical activity. Thus, some participants may have felt discouraged by their lack of weight loss during the intervention, which has been highlighted as a predictor of attrition in previous obesity-related studies ${ }^{(14,34)}$.

Our characterisation of dropouts $v$. completers is broadly similar to previous lifestyle-based intervention studies. We found that younger age and higher BMI were strong predictors of greater attrition, which confirms previous findings ${ }^{(35,36)}$. Older individuals may be more interested in sustained participation due to increased health concerns and heightened perceived susceptibility to disease. Obese individuals are often characterised by poor diet and low levels of physical activity ${ }^{(37)}$, which may make lifestyle changes challenging. In contrast to an earlier report that individuals from lower socio-economic status are more likely to drop out of lifestyle interventions $^{(8)}$, we found no differences in attrition between occupation groups. This may be due to the personalised nature of the Food4Me intervention; recent research suggests that lifestyle interventions may be more effective in individuals with low socio-economic status if they use tailored, or personalised, advice based on information about individual physical condition such as being overweight or having high cholesterol concentration ${ }^{(38)}$. However, it may also be due to the higher socio-economic status of our participants and that our measure of socioeconomic status was limited to occupation. We did not identify any difference in health and disease status between completers and those who dropped out. Although some associations between attrition and health-related characteristics have been observed ${ }^{(39)}$, results have been inconsistent ${ }^{(40)}$.

Inter-country differences in attrition observed in our analyses may partly be explained by the timing of the interventions. Ireland and the UK were the first centres to commence the Food4Me intervention and so the higher dropout rates (although not significant for the UK) may be a result of initial teething problems in delivering the intervention, such as responding to queries from participants, which were resolved when the other centres initiated recruitment. There is no obvious explanation for the significantly lower dropout rate in The Netherlands, but may have been due to centre-to-centre variation in the perseverance of researchers. Attrition was similar for control and PN intervention arms; however, individuals were more likely to drop out if they were in the high intensity feedback group. The burden associated with the higher number of occasions that participants were contacted to complete their FFQ and provide their phenotypic data between baseline and month 3 may explain these results more than receiving more frequent PN feedback per se. Alternatively, although individuals in the high intensity group had access to online discussion forums, personalised recipes and additional PA advice, while those in the low intensity group did not, the perceived value to participants of the more frequent feedback may not have been sufficient to outweigh the added burden of completing extra questionnaires. As a result, further consideration of the nature and frequency of such feedback may be important for future study designs.

Our study is the first Internet-based PN study to characterise dropouts based on their dietary habits. Although many studies have associated sociodemographic characteristics, such as age and social class, with attrition $^{(8,15)}$, behavioural determinants, such as reasons for participation and dietary habits, require further elucidation $^{(9,41)}$. Improved understanding of these factors may help in tailoring interventions to the needs of participants $^{(10)}$ and hence reduce dropout. Furthermore, a systematic review of predictors of dropout in weight-loss interventions reported that poor eating habits were associated with higher dropout rates ${ }^{(9)}$. We found that participants were more likely to drop out if they skipped meals and if they ate their main meal away from home, suggesting that it may be more difficult for individuals with these dietary habits to comply with a PN intervention. As a result, future design of $\mathrm{PN}$ advice would benefit from incorporating eating behaviour characteristics. Participants in the Food4Me study were also less likely to drop out if they reported that they often ate healthily, did not have to consciously think about eating healthily and had lower PUFA and higher salt intakes. These findings are consistent with previous studies, where healthier individuals are more interested and willing to participate in and complete lifestyle interventions ${ }^{(9)}$. However, participants in the Food4Me Proof-of-Principle study were broadly representative of the European population in terms of obesity prevalence and dietary adequacies, and so would benefit from improved diet and $\mathrm{PA}^{(42)}$. Although psychological determinants of attrition have been studied ${ }^{(43,44)}$, the role of influences such as life stress, motivation and perceived self-efficacy on attrition in a PN intervention is poorly understood $^{(45)}$.

The present study had a number of strengths. The Food4Me Proof-of-Principle study included a large number of participants from seven different European countries. By collecting information on sociodemographics, anthropometrics, PA and dietary intakes, as well as information on dietary habits, we had 
a comprehensive overview of the characteristics of participants who dropped out of an Internet-based PN intervention.

A limitation of the present study is that psychological determinants of attrition were not investigated. Psychological constructs, such as perceived self-efficacy, may affect behaviour change and thus attrition. For example, an individual with a low perceived self-efficacy may be less likely to follow dietary advice and thus be less likely to remain in a dietary intervention ${ }^{(46)}$. However, as a proofof-principle study, assessment of psychological determinants was not within the scope of the present study. As a result, the present findings should be interpreted with the understanding that psychological constructs may have played a role in determining attrition and further research into these specific determinants is warranted. A potential limitation of the study is that our data were self-reported via the Internet, which may have introduced measurement error. However, the validity of Internet-based, selfreported anthropometric data is high $^{(47)}$ and has been confirmed in the present study ${ }^{(27)}$. Dietary intakes were estimated by an FFQ, which is known to be subject to misreporting error ${ }^{(48)}$, but this was minimised by validating our FFQ against a $4 \mathrm{~d}$ weighed food record ${ }^{(20)}$. Occupations were not asked for the purpose of socio-economic status and so the specificity of the classification of the occupations could not always be guaranteed. Our study participants were predominantly Caucasian so further research among wider ethnicity groups is required to generalise our findings to other populations.

Our findings suggest that future PN interventions would benefit from strategies designed to sustain compliance from younger participants and those who are obese. Importantly, future PN interventions should consider dietary habits, such as the frequency of meal skipping and eating main meals away from home, and psychological characteristics of their participants to develop strategies to help such participants remain in the study. In addition, our finding of a higher dropout rate among those completing more FFQ and receiving more frequent feedback suggests that the extra burden of completing additional questionnaires may be detrimental to their compliance with the intervention.

\section{Conclusions}

Attrition in the Food4Me PN intervention study delivered via the Internet was close to the average for other lifestylebased interventions. There was no difference in dropout rate between those randomised to the Control group (generalised dietary advice) and those randomised to receive $\mathrm{PN}$ advice. However, more frequent data collection and PN feedback and behavioural barriers to healthy eating were strong determinants of attrition. Future PN interventions would benefit from improved strategies to minimise dropouts among younger and obese individuals. Findings from the present study will be of value to researchers who wish to design and implement Internet-delivered $\mathrm{PN}$ interventions which have considerable potential to deliver improved lifestyle behaviours and, therefore, benefits for public health.

\section{Acknowledgements}

Financial support: This work was supported by the European Commission under the Food, Agriculture, Fisheries and Biotechnology Theme of the 7th Framework Programme for Research and Technological Development (265494). The European Commission had no role in the design, analysis or writing of this article. Conflict of interest: None of the authors had a personal or financial conflict of interest. Authorship: K.M.L. and C.C.-M. are joint first authors. Author responsibilities were as follows: Y.M., I.T., C.A.D., E.R.G., L.B., J.A.L., J.A.M., W.H.M.S., H.D., M.G. and J.C.M. contributed to the research design. J.C.M. was the Food4Me Proof-of-Principle study leader. C.C.-M., C.F.M.M., H.F., C.B.O., C.W., A.L.M., R.F., S.N.-C., R.S.-C., S.K., L.T., C.P.L., A.S., M.C.W., E.R.G., L.B. and J.C.M. contributed to developing the standardised operating procedures for the study. C.C.-M., S.N.-C., R.S.-C., C.W., C.B.O., H.F., C.F.M.M., A.L.M., R.F., S.K., L.T., C.P.L., A.S., M.C.W. and J.C.M. conducted the intervention. C.C.-M., C.F.M.M. and W.H.M.S. contributed to physical activity measurements. K.M.L. and C.C.-M. wrote the paper and performed the statistical analysis for the manuscript. All authors contributed to a critical review of the manuscript during the writing process. All authors approved the final version to be published. Ethics of human subject participation: This study was conducted according to the guidelines laid down in the Declaration of Helsinki and all procedures involving human subjects/patients were approved by the Research Ethics Committees at each University or Research Centre delivering the intervention. The Food4Me trial was registered as a randomised controlled trial (NCT01530139) at Clinicaltrials.gov. All participants expressing an interest in the study were asked to sign online consent forms at two stages in the screening process. These consent forms were automatically directed to the local study investigators to be counter-signed and archived.

\section{Supplementary material}

To view supplementary material for this article, please visit http://dx.doi.org/10.1017/S1368980016002020

\section{References}

1. Ng M, Fleming T, Robinson M et al. (2014) Global, regional, and national prevalence of overweight and obesity in children and adults during 1980-2013: a systematic analysis for the Global Burden of Disease Study 2013. Lancet 384, 766-781 
2. World Health Organization (2014) Global Status Report on Noncommunicable Diseases 2014. Geneva: WHO.

3. Sepúlveda J \& Murray C (2014) The state of global health in 2014. Science 345, 1275-1278.

4. Hood L \& Friend SH (2011) Predictive, personalized, preventive, participatory (P4) cancer medicine. Nat Rev Clin Oncol 8, 184-187.

5. Celis-Morales C, Lara J \& Mathers JC (2015) Personalising nutritional guidance for more effective behaviour change. Proc Nutr Soc 74, 130-138.

6. Nielsen DE \& El-Sohemy A (2014) Disclosure of genetic information and change in dietary intake: a randomized controlled trial. PLOS ONE 9, e112665.

7. Livingstone KM, Celis-Morales C, Navas-Carretero S et al. (2016) Effect of an Internet-based, personalized nutrition randomized trial on dietary changes associated with the Mediterranean diet: the Food4Me Study. Am J Clin Nutr (Epublication ahead of print version).

8. Roumen C, Feskens EJM, Corpeleijn E et al. (2011) Predictors of lifestyle intervention outcome and dropout: the SLIM study. Eur J Clin Nutr 65, 1141-1147.

9. Moroshko I, Brennan L \& O'Brien P (2011) Predictors of dropout in weight loss interventions: a systematic review of the literature. Obes Rev 12, 912-934.

10. Huisman S, Maes S, De Gucht VJ et al. (2010) Low goal ownership predicts drop-out from a weight intervention study in overweight patients with type 2 diabetes. Int $J$ Behav Med 17, 176-181.

11. Bennett GA \& Jones SE (1986) Dropping out of treatment for obesity. J Psychosom Res 30, 567-573.

12. Dalle Grave R, Calugi S, Molinari E et al. (2005) Weight loss expectations in obese patients and treatment attrition: an observational multicenter study. Obes Res 13, 1961-1969.

13. Davis M \& Addis M (1999) Predictors of attrition from behavioral medicine treatments. Ann Behav Med 21, 339-349.

14. Colombo O, Ferretti VV, Ferraris C et al. (2014) Is drop-out from obesity treatment a predictable and preventable event? Nutr J 13, 13.

15. Groeneveld I, Proper K, van der Beek A et al. (2009) Factors associated with non-participation and drop-out in a lifestyle intervention for workers with an elevated risk of cardiovascular disease. Int J Behav Nutr Phys Act 6, 80.

16. Williams G, Hamm MP, Shulhan J et al. (2014) Social media interventions for diet and exercise behaviours: a systematic review and meta-analysis of randomised controlled trials. BMJ Open 4, e003926.

17. Shuger S, Barry V, Sui X et al. (2011) Electronic feedback in a diet- and physical activity-based lifestyle intervention for weight loss: a randomized controlled trial. Int J Behav Nutr Phys Act 8, 41 .

18. Celis-Morales C, Livingstone KM, Marsaux CFM et al. (2015) Design and baseline characteristics of the Food4Me study: a web-based randomised controlled trial of personalised nutrition in seven European countries. Genes Nutr 10, 450.

19. Forster H, Fallaize R, Gallagher C et al. (2014) Online dietary intake estimation: the Food $4 \mathrm{Me}$ food frequency questionnaire. J Med Internet Res 16, e150.

20. Fallaize R, Forster H, Macready AL et al. (2014) Online dietary intake estimation: reproducibility and validity of the Food4Me food frequency questionnaire against a 4-day weighed food record. J Med Internet Res 16, e190.

21. Food Standards Agency (2002) McCance and Widdowson's The Composition of Foods, sixth summary edition ed. Cambridge: Royal Society of Chemistry.

22. Henry CJK (2005) Basal metabolic rate studies in humans: measurement and development of new equations. Public Health Nutr 8, 1133-1152.

23. Institute of Medicine (2005) Dietary Reference Intakes for Energy, Carbohydrate, Fibre, Fat, Fatty Acids, Cholesterol,
Protein, and Amino Acids. http://www.nap.edu/openbook. php?isbn=0309085373 (accessed March 2015).

24. Institute of Medicine (2011) Dietary Reference Intakes Tables and Applications. http://www.iom.edu/Activities/ Nutrition/SummaryDRIs/DRI-Tables.aspx (accessed March 2015).

25. World Health Organization (2007) Protein and Amino Acid Requirements in Human Nutrition. Report of a Joint WHO/ FAO/UNU Expert Consultation. WHO Technical Report Series no. 935. Geneva: WHO.

26. World Health Organization \& Food and Agriculture Organization of the United Nations (2010) Report of an expert consultation on fats and fatty acids in human nutrition. http://www.who.int/nutrition/publications/ nutrientrequirements/fatsandfattyacids_humannutrition/en/ (accessed March 2016).

27. Celis-Morales C, Livingstone K, Woolhead C et al. (2015) How reliable is internet-based self-reported identity, sociodemographic and obesity measures in European adults? Genes Nutr 10, 476.

28. European Commission (2015) European skills, competences, qualifications and occupations. https://ec.europa.eu/ esco/web/guest/hierarchybrowser/-/browser/Occupation (accessed April 2015).

29. European Commission (2015) Mean annual earnings by sex, age and occupation. http://ec.europa.eu/eurostat/web/ products-datasets/-/earn_ses_agt28 (accessed March 2015).

30. Clark F, Jackson J, Carlson M et al. (2012) Effectiveness of a lifestyle intervention in promoting the well-being of independently living older people: results of the Well Elderly 2 Randomised Controlled Trial. I Epidemiol Community Health 66, 782-790.

31. Neve M, Morgan PJ, Jones PR et al. (2010) Effectiveness of web-based interventions in achieving weight loss and weight loss maintenance in overweight and obese adults: a systematic review with meta-analysis. Obes Rev 11, 306-321.

32. Tate DF, Wing RR \& Winett RA (2001) Using internet technology to deliver a behavioral weight loss program. JAMA 285, 1172-1177.

33. Harvey-Berino J, Pintauro S, Buzzell P et al. (2004) Effect of internet support on the long-term maintenance of weight loss. Obes Rev 12, 320-329.

34. Grossi E, Dalle Grave R, Mannucci E et al. (2006) Complexity of attrition in the treatment of obesity: clues from a structured telephone interview. Int J Obes (Lond) 30, 1132-1137.

35. Wanner M, Martin-Diener E, Bauer G et al. (2010) Comparison of trial participants and open access users of a web-based physical activity intervention regarding adherence, attrition, and repeated participation. J Med Internet Res 12, e3.

36. Susin N, Boff RdM, Ludwig MWB et al. (2015) Predictors of adherence in a prevention program for patients with metabolic syndrome. J Health Psychol (Epublication ahead of print version).

37. Mesas AE, Guallar-Castillón P, León-Muñoz LM et al. (2012) Obesity-related eating behaviors are associated with low physical activity and poor diet quality in Spain. J Nutr $\mathbf{1 4 2}$, $1321-1328$

38. Bukman AJ, Teuscher D, Feskens EJM et al. (2014) Perceptions on healthy eating, physical activity and lifestyle advice: opportunities for adapting lifestyle interventions to individuals with low socioeconomic status. BMC Public Health 14, 1036.

39. Graffagnino CL, Falko JM, La Londe M et al. (2006) Effect of a community-based weight management program on weight loss and cardiovascular disease risk factors. Obesity (Silver Spring) 14, 280-288.

40. Gripeteg L, Karlsson J, Torgerson J et al. (2010) Predictors of very-low-energy diet outcome in obese women and men. Obes Facts 3, 159-165. 
41. Davis MJ \& Addis ME (1999) Predictors of attrition from behavioral medicine treatments. Ann Behav Med 21, 339-349.

42. Livingstone K, Celis-Morales C, Navas-Carretero S et al. (2016) Profile of European adults interested in internet-based personalised nutrition: the Food4Me study. Eur J Nutr 55, 759-769.

43. Post A, Gilljam H, Bremberg S et al. (2012) Psychosocial determinants of attrition in alongitudinal study of tobacco use in youth. Scientific World Journal 2012, 654030 .

44. Cochrane G (2008) Role for a sense of self-worth in weightloss treatments: helping patients develop self-efficacy. Can Fam Physician 54, 543-547.
45. Mutsaerts MAQ, Kuchenbecker WKH, Mol BW et al. (2013) Dropout is a problem in lifestyle intervention programs for overweight and obese infertile women: a systematic review. Hum Reprod 28, 979-986.

46. Schwarzer R \& Renner B (2000) Social-cognitive predictors of health behavior: action self-efficacy and coping selfefficacy. Health Psychol 19, 487-495.

47. Pursey K, Burrows LT, Stanwell P et al. (2014) How accurate is web-based self-reported height, weight, and body mass index in young adults? J Med Internet Res 16, e4.

48. Macdiarmid J \& Blundell J (1998) Assessing dietary intake: who, what and why of under-reporting. Nutr Res Rev 11, 231-253. 